\title{
Research Paper \\ Culture and Nutrition Knowledge, and Their Relationship With Food Insecurity Among Rural Households in Kermanshah County, Iran
}

\author{
*Heydar Gholizadeh ${ }^{1}$, Zohreh Rostamian Motlagh², Mohammad Badsar ${ }^{1}$, Ali Shams ${ }^{1}$
}

1. Assistant Professor, Department of Agricultural Extension, Communication and Rural Development, Faculty of Agriculture, University of Zanjan, Zanjan, Iran. 2. MSc., Department of Agricultural Extension, Communication and Rural Development, Faculty of Agriculture, University of Zanjan, Zanjan, Iran.

\begin{tabular}{|l|l|}
\hline $\begin{array}{c}\text { Use your device to scan } \\
\text { and read the artice online }\end{array}$ \\
Their Relationship With Food Insecurity Among Rural Households in Kermanshah County, Iran (Persian)]. Journal of Rural \\
Research, 8(1), 54-67. http://dx.doi.org/10.21859/jjr-08014 \\
doi: : http://dx.doi.org/10.21859/jjr-08014
\end{tabular}

Received: 21 Aug. 2016

Accepted: 25 Dec. 2016

Key words:

Nutrition culture,

Nutrition knowl-

edge, Food inse-

curity, Mediating

effect, Kermanshah

county

\begin{abstract}
A BSTRACT
Food security is a key element in individuals' mental, psychological, and physical health. Meanwhile, the food insecurity is a serious problem, especially in the underdeveloped and developing countries. Although availability, access, utilization, and stability are noted in the food security definitions, it seems that there are factors beyond the framework, which affect food security. Food insecurity depends on the consumption behavior and its underlying factors. In the meantime, the feeding pattern and habits, derived from the culture and knowledge of nutrition, also affect human health. The current study, in terms of the nature, is a quantitative research, and in terms of purpose is an applied research. The information was collected by a 2-part questionnaire including the researcher-made questionnaire and the household food insecurity access scale (HFIAS). A number of 258 heads of rural households was selected as the study sample from 11 villages in Kermanshah County, Iran, using the multistage sampling method. The results showed that while more than $58.5 \%$ of the rural households were in food security or mild food insecurity status, about $14.7 \%$ of the households faced with severe food insecurity. Results of structural equation modeling showed that nutrition knowledge had a negative and significant impact on food insecurity. The results also showed that nutrition knowledge had an indirect effect on food insecurity through nutrition culture, which should be considered in the food security studies.
\end{abstract}

\section{Extended Abstract}

\section{Introduction}

$\mathbf{F}$

ood security is a key element in individuals' mental, psychological and physical health, and it is also considered as the cornerstone of a developed society. Food security occurs when the nutritional conditions and economics, social, cultural, and sanitary indicators together reach desirable stable level. However, the food insecurity is a serious problem in the world. Although availability, access, utilization, and stability are noted in the food security definitions, there seems to be some factors beyond the framework, which affect food security.

Food insecurity and malnutrition have undesirable consequences for health and development and they are major obstacles to achieve the development goals. Culture, as one of the predisposing factors of food security, affects all aspects of human life. Hence, it could be said that ideas and beliefs, behaviors, customs and feeding habits are affected by the cultural framework and the context of a society. In the meantime, the feeding pattern and hab-

\section{* Corresponding Author:}

Heydar Gholizadeh, PhD

Address: Department of Agricultural Economics, Communication and Rural Development, Faculty of Agriculture, University of Zanjan, Zanjan, Iran Tel: +98 (935) 5957362

E-mail: hgholizadeh@znu.ac.ir 
its, derived from the culture and knowledge of nutrition, also affect human health. In fact, one of the important aspects which clearly shows the impact of culture on human health is nutrition and food habits. The current study aimed at investigating the relationship between the culture and nutrition knowledge with food insecurity among the rural household of Kermanshah County, Iran.

\section{Methodology}

The current study was qualitative in terms of nature and applied in terms of purpose. The data were collected by a 2-part questionnaire including a researcher-made questionnaire and the household food insecurity access scale (HFIAS). The researcher-made questionnaire consisted of questions on rural households' life conditions, their food culture, and also nutritional knowledge. Social and economic data were collected mostly through the demographic variables. Nutrition culture was studied in 4 sections including underlying beliefs, feeding pattern, behavior pattern, and personal interest based on a Likert scale. Nutrition knowledge was studied based on 6 items. Moreover, HFIAS was used to assess the level of food insecurity in rural households. A number of 258 heads of rural households were selected as the study sample from 11 villages in 4 rural districts in Kermanshah County, using the multistage sampling method.

The face and content validity of the questionnaire was approved by a panel of experts (faculty members at Zanjan University, Zanjan, Iran). A pilot study was conducted to determine the reliability of the instrument. The Cronbach's alpha coefficient for HFIAS items, and nutrition culture and nutritional knowledge sections was 0.89 , 0.76 , and 0.74 , respectively, indicating good reliability. Data analysis was conducted by SPSS version 20 and AMOS version 20 software.

\section{Results}

Descriptive statistics significantly showed that $12.6 \%$ of the total sample households were female-headed, and $95.3 \%$ of them were married. In terms of education level, while $26 \%$ of them were illiterate, only $4.3 \%$ had academic education. The mean age of respondents was 50.5 years, and $60.3 \%$ of them had 46 years and over. The obtained results showed that about $14.7 \%$ of the subjects had severe food insecurity and more than $26.7 \%$ had moderate food insecurity. However, more than $58.5 \%$ of rural households were in food security or mild food insecurity status (15.1\% and 43.4\%, respectively).
To test the impact of culture and nutrition knowledge on the food insecurity, the structural equation model was estimated. The result of assessing direct structural model fits indicated that the model fit the data with $\chi^{2}(12)=25.114$, $\mathrm{P}=0.002, \chi^{2} / \mathrm{df}=2.093 ; \mathrm{GFI}=0.973, \mathrm{CFI}=0.985, \mathrm{IFI}=0.985$, $\mathrm{RMSEA}=0.066$. The Goodness-of-fit indices of structural model indicated that the GFI, CFI, and IFI significantly passed their cutoff value (0.90). In addition, the RMSEA was 0.066 , which fell between the recommended range of acceptability (between 0.03 and 0.08 ). The result showed nutrition knowledge as an independent variable, and explained about $31 \%$ of the variance of food insecurity. Based on the model, nutrition knowledge had a negative and significant impact on food insecurity.

To evaluate the effect of culture, multipurpose bootstrapping method was implemented, using Amos version 20 graphic software. Initially, the supposed mediator conceptual model was designed; then, based on the statistical sample, suggested by Hayes, 5000 subjects were recruited and replaced by $95 \%$ confidence interval through multiple sub-sampling by replacement based on the original data from 254 of the rural households. The result of bootstrapping this model showed that the indirect effect of food culture through nutrition knowledge was significant $(\beta=-0.051, P=0.001)$. As a result, it could be said that food culture had a mediating effect on the relationship between nutrition knowledge and food insecurity status. In summary, nutrition knowledge had the highest direct effect $(\beta=-0.508)$ and the total effect on the rural food insecurity $(\beta=-0.56)$.

\section{Discussion}

The results showed that only $15.14 \%$ of the households were in completely food security status and $84.86 \%$ of them faced various degrees of food insecurity. These results were comparable with the findings of Saadi et al., indicating that $85 \%$ of Gorveh, Iran, households faced the kind of food insecurity. Also, Salarkia et al. and Saadi and Vahdat-Moaddab reported $79 \%$ and $75 \%$ of food insecurity between households in Varamin and in femaleheaded households in Razan, Iran, respectively.

In the current study, nutrition knowledge was considered in 2 ways involving the awareness of useful foods and harmful foods, and the consumption of such foods. Results of structural equation modeling showed that nutrition knowledge had a negative and significant impact on food insecurity. The results also indicated that despite awareness of households about the useful foods (such as dairy, vegetables, and fruits as a main part of food pyramid), and also harmful foods (such as fatty, sugary, and 
salty foods) they had a poor performance in the application of knowledge in their nutrition. It was either due to the low economic status of households or because of their taste or interest in harmful foods. The result also showed that nutrition knowledge had an indirect effect on food insecurity through nutrition culture, which should be considered in the food security studies.

\section{Conclusion}

Accordingly, as the people eating behavior is influenced by nutritional knowledge, educating people, especially heads of households about the hazards of fatty, salty, and sweet foods as well as fast food, as well as providing solutions to replace healthy foods instead of these harmful foods, can improve the nutritional knowledge of families. In addition, nutrition education can be implemented in schools in order to create and establish correct eating habits of children. Another appropriate solution for training and development of a healthy diet in a society is taking advantage of the mass media, especially the visual media.

\section{Acknowledgments}

This research was extracted from the MA thesis of the second author, in the Department of Agricultural Extension, Communication and Rural Development, Faculty of Agriculture, University of Zanjan, Zanjan, Iran.

\section{Conflict of Interest}

The authors declared no conflicts of interest. 


\title{
فرهنَّ و دانش تغذيهاي و ارتباط آن با نامنى غذايى در خانوارهاى روستايى شهرستان كرمانشاه
}

\author{
"حيدر قلىزاده'، زهره رستميان مطلق'، محمد بادسار'، على شمس'

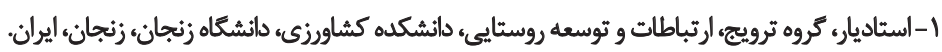

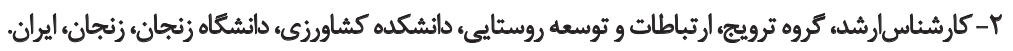

\begin{abstract}
Q

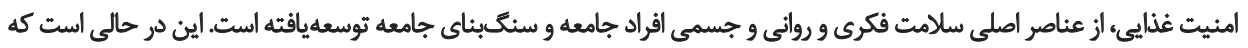

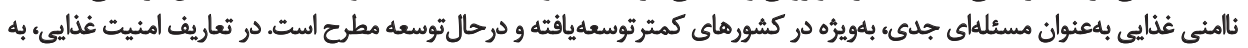

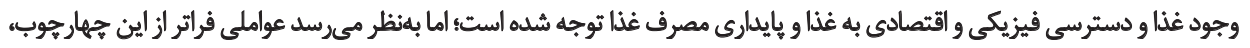

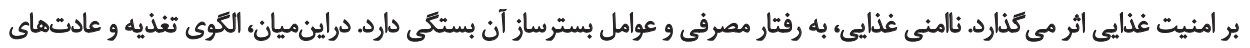

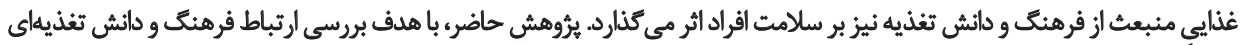

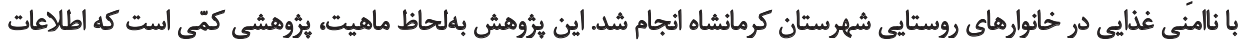

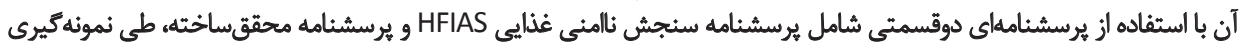

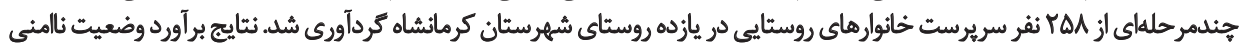

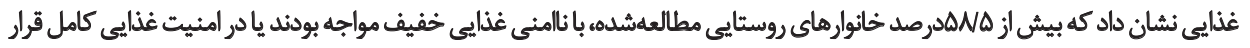

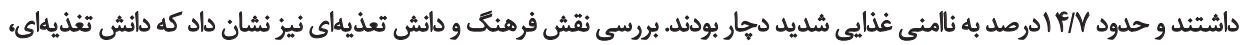

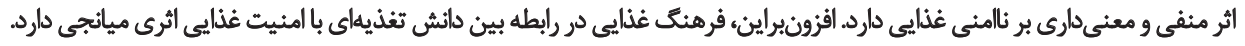

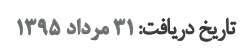

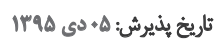

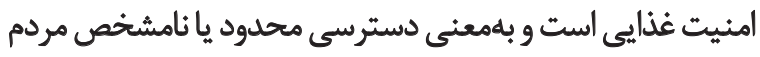

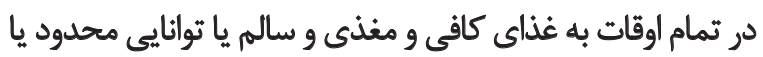

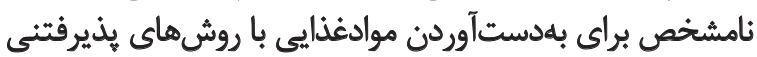

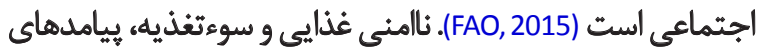
نامطلوبى براى سلامت و توسعه بهدنبال دارد (Reichwage, 2010).

در بُعد خانوار، مهمترين عامل تعيين كنينده امنيت غذايتي، توانايى

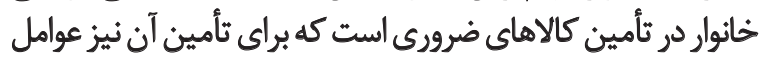

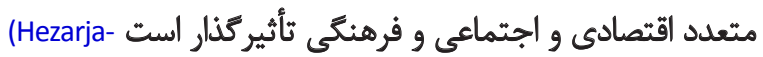
توين (ribi \& Alizadeh Aghdam, 2013)

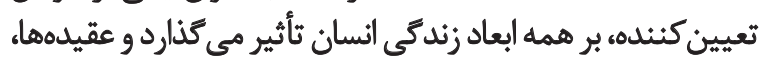

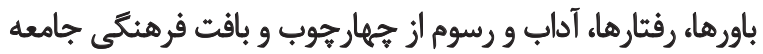

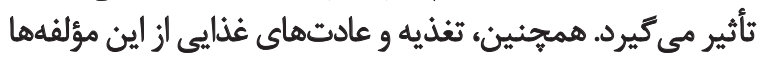

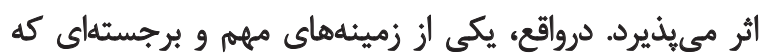

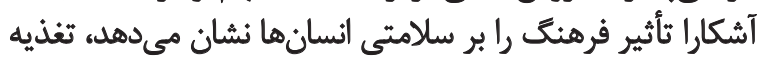

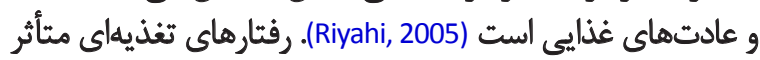

دسترسى به غذاى كافى و مطلوب و سلامت تغذيهاي، از

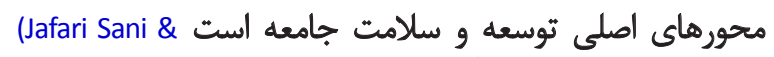

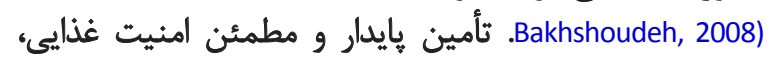

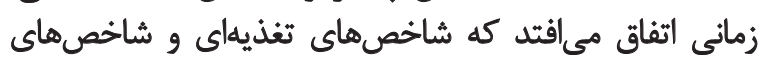

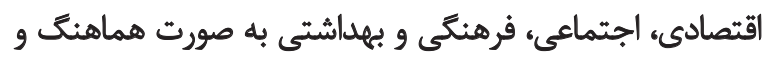

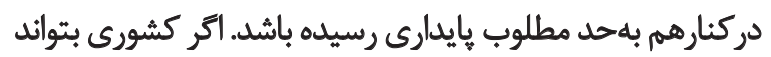

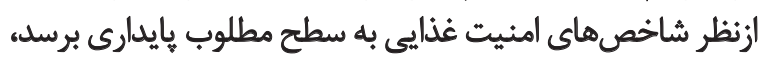

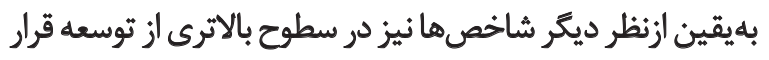
مي ميرد (Rostami, Shahmoradi, \& Baghaie, 2015).

درحقيقت، امنيت غُذايى سنكبناى جامعه توسعهيافته و

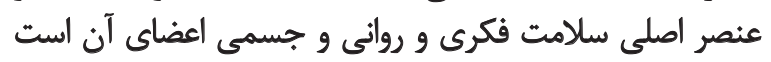

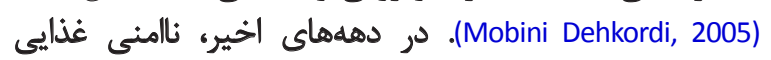

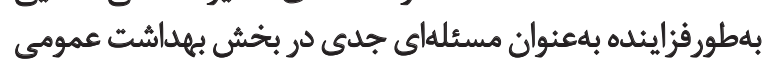

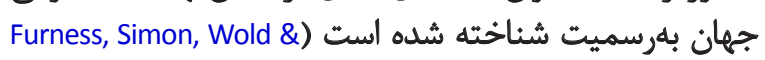
آمارها نشان مى شهد كه درحال حاضر، حدود (Anderson, 2004) 


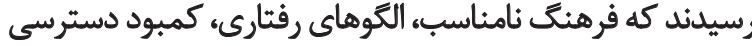

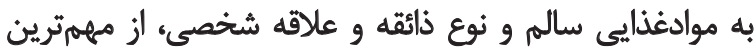

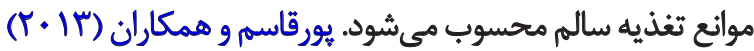

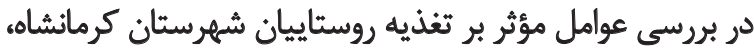

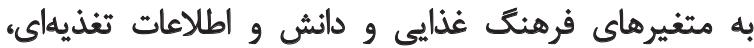
بهعنوان يكى از عوامل مؤثر بر الكوى مصرف و و مقدي مقدار مصرف

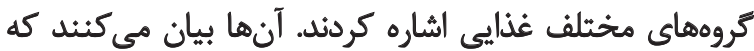

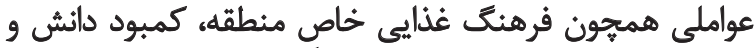

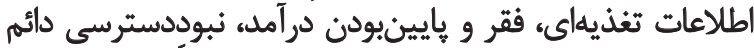

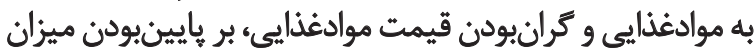

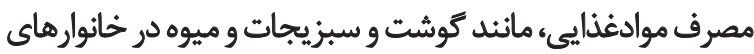

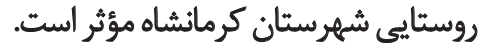

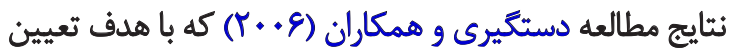

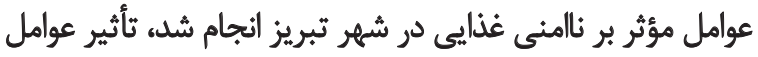

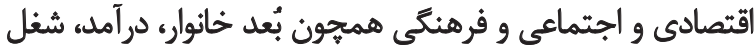

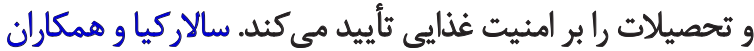
(Y. II)

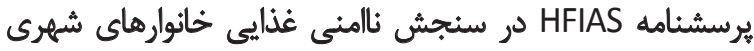

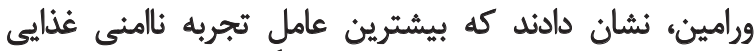

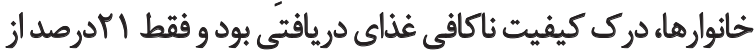
خانوارهاى ورامين در طبقه امن غذايى قرار داشتئد. دراني انارستاني دئي

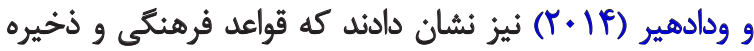

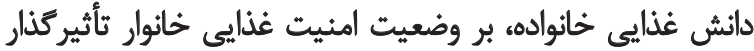

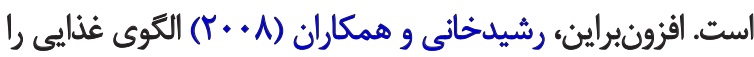

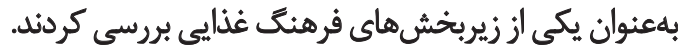
بهدليل ماهيت بينرشتهاي اين مسئله و لزوم دركيرشدن

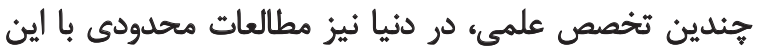

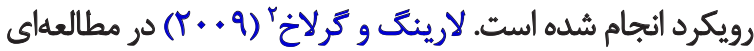

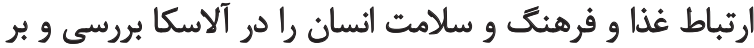

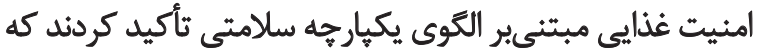

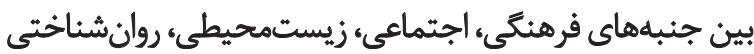

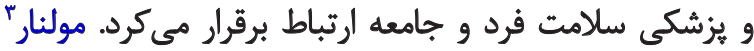

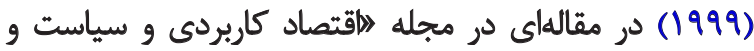

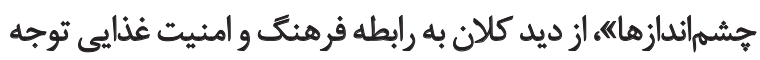

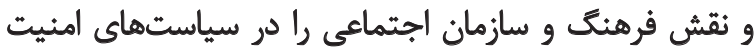

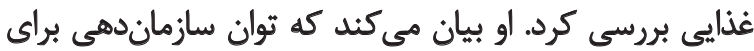

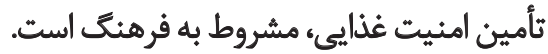

بهدليل دشوارى شناسايي و سنجش متغير هايي كه بازگتوكنئه

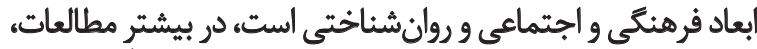

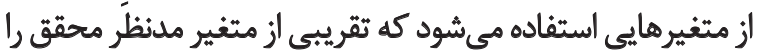

2. Loring \& Gerlach

3. Molnar

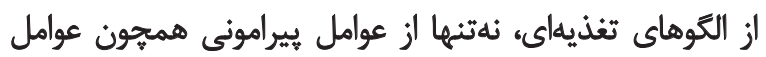
مختلف بيولوزيكى، محيطى، فرهنكي، اقتصادى و اجتماعى تأثير

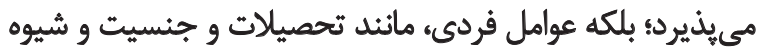

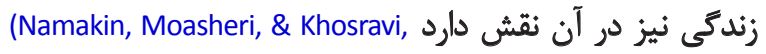

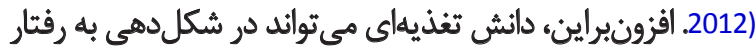

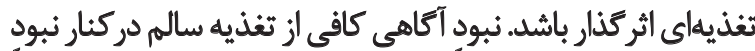

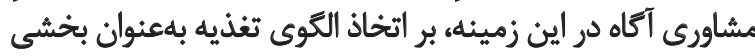

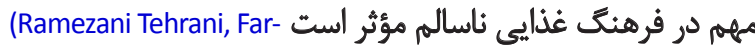
ahmand, Amiri, Ghanbarian, \& Azizi, 2011a).

تبيين نقش فرهنگ و دانش تغذيهاى، در شكل تيرى الكوها

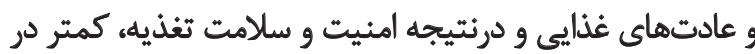

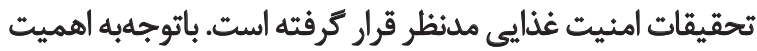

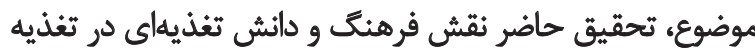

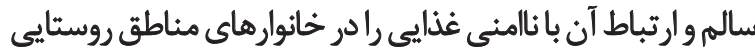

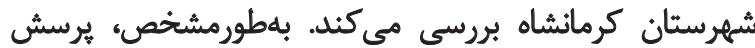

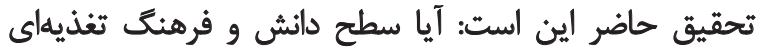

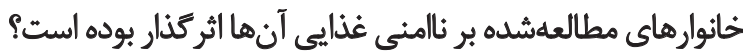

\section{مرورى بر أدبيات موضوع}

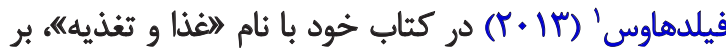

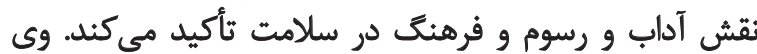

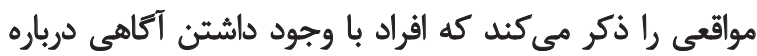

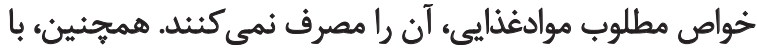

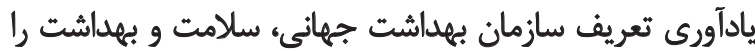

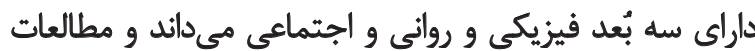

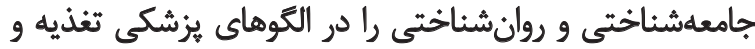

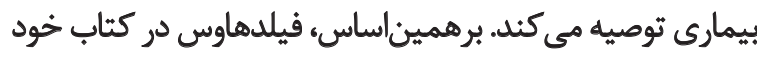

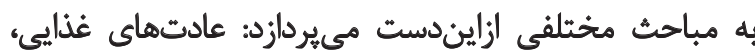

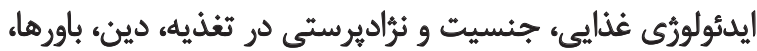

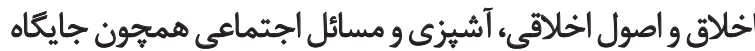
و مثزلت اجتماعى و حتى روانشئاسى انتخاب غذانيا.

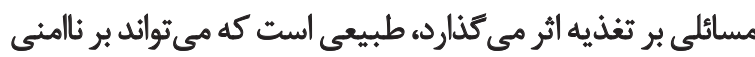

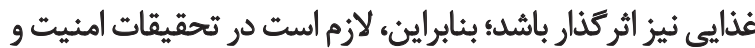

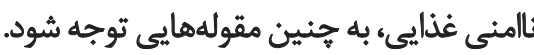

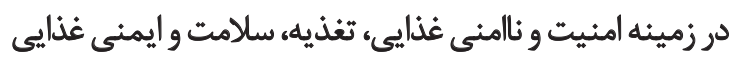

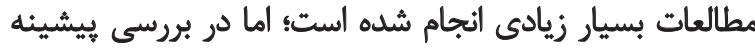

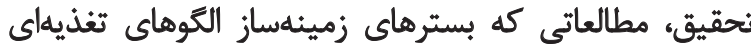

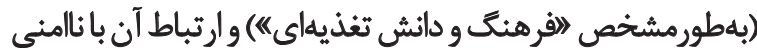

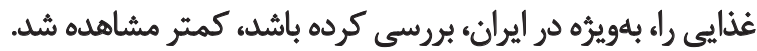

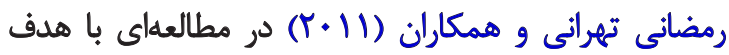

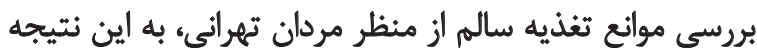

1. Fieldhouse 
r. بروسى ارتباط بين فرهنك تغذيهاي و نامنى غذاييى

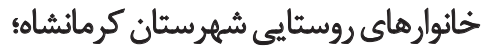

"r. بررسى ارتباط بين دانش تغذيهاي و ناامنى غذاييى خانوارهاي روستايي شهرستان كرمائشاه.

\section{روش ش رشناسى تحقيق}

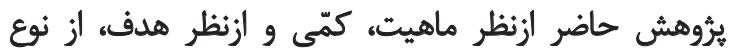

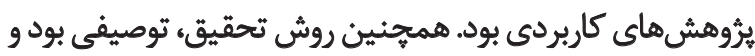

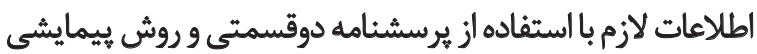

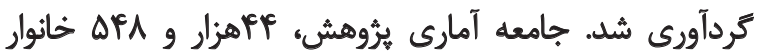

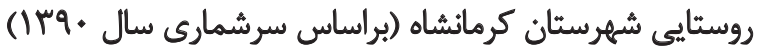

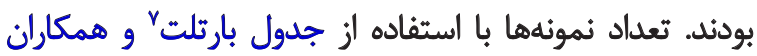

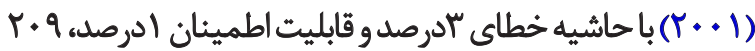

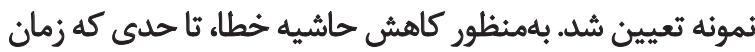

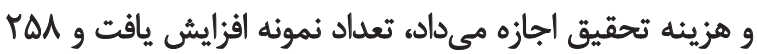

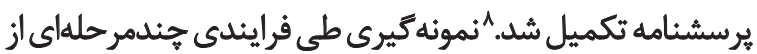

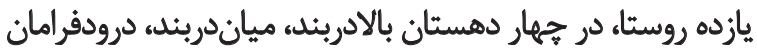

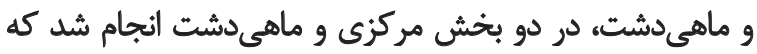

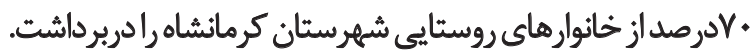

يرسشنامه شامل دو بخش بود: بخش اول، مربوطبه اطلاعات

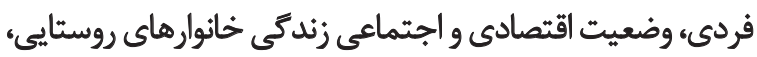

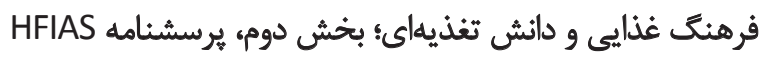

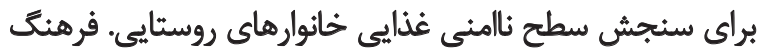

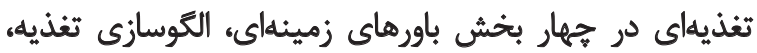

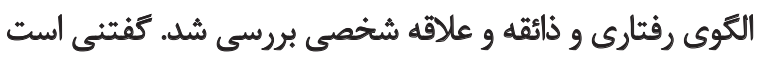

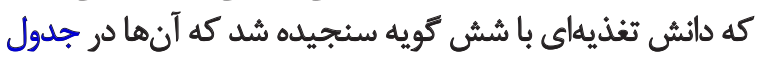
شماره الو آورده شده است باست.

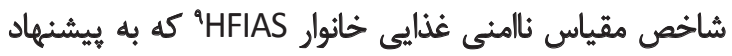

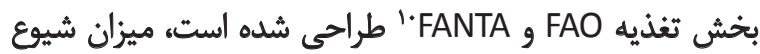

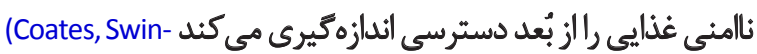

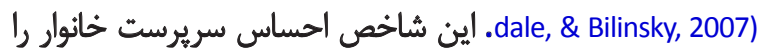

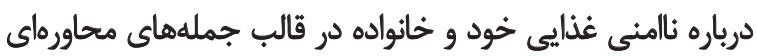

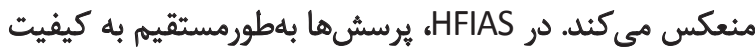

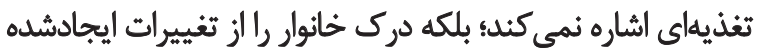

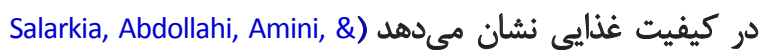

.(Eslami Amirabadi, 2011

براى سنجش روايى ظاهرى و محتوايى يرسشنامه، از ديدكاهها

7. Bartlett et al.

1. با اين افزايش تعداد نمونه انتخابى، انتظار مىرود حاشيه خطا به حدود ه/ كدرصد

$$
\text { كاهش يافته باشد. }
$$

9. Household Food Insecurity Access Scale (HFIAS)

10. Food and Nutrition Technical Assistance Project (FANTA)
ار ائه دهد. متغيرهاى جمعيتشناختى، شكل غالب اين مفاهيم رادر

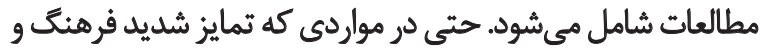

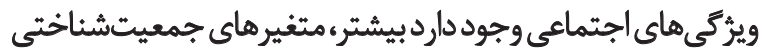

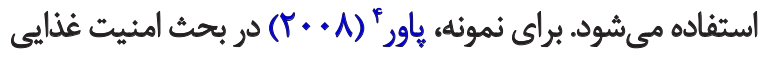

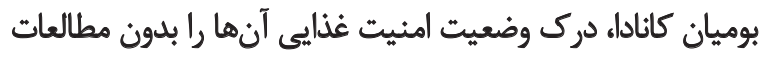

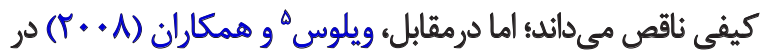

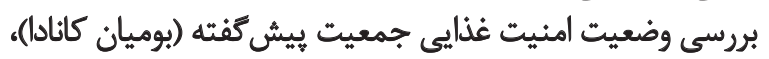

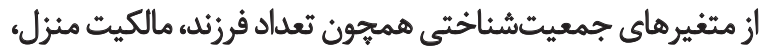
سطح تحصيلات و سطح درآمد بهره مي مئيرند.

يكى از رويكردهاى مطالعات امنيت غذايى، ثلاش براي براي

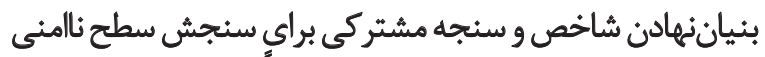

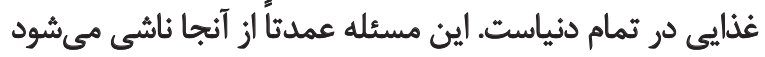

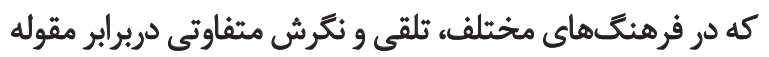

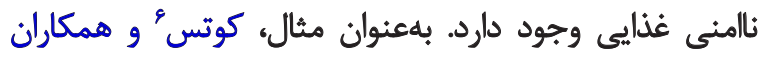

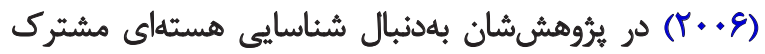

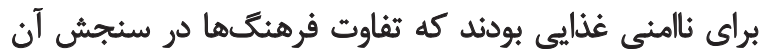

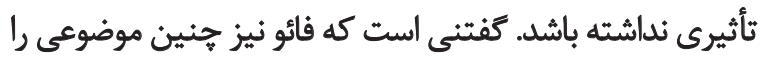
تعقيب مى كند (Ballard, Kepple, \& Cafiero, 2013).

در زمينه امنيت غذايى در مناطق روستايى استان كرمانشاه،

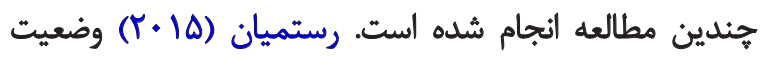

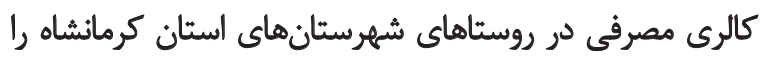

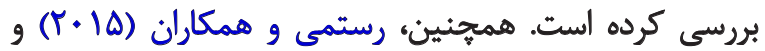

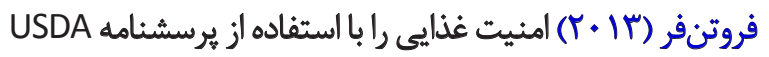

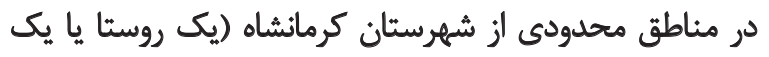

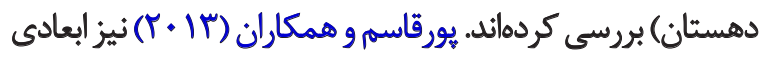

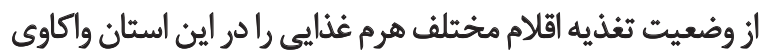

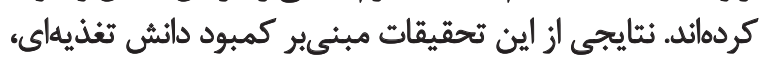

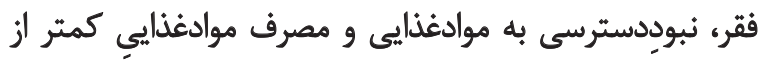

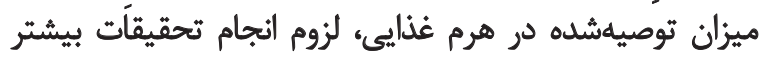

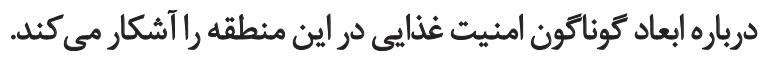

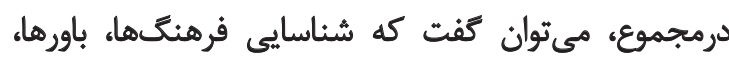

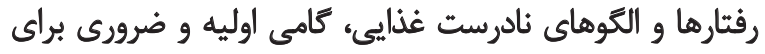

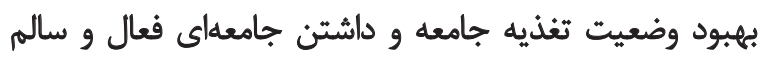

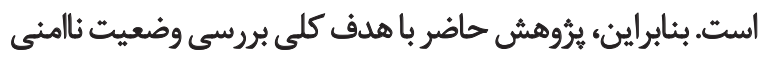

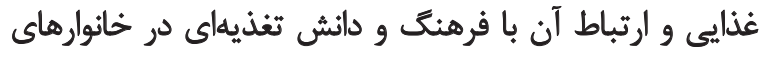

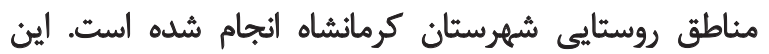
يُروهش اهداف اختصاصى زير را دنبال مي كنداند 1. بررسى و سنجش سطح نامنى غذايى خانوار هاى روستايى

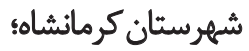

4. Power

5. Willows et al.

6. Coates et al. 
جدول ا. كويههاي سنجش شاخصهاي فرهنت و دانش تغذيهاي

\section{فر هنى تغذيهاى}

از نظر من، مصرف غذاهاى آماده، مانند سوسيس و كالباس نشانه تجدد و امروزىبودن است.

از نظر خودم يا اطرافيانهه استفاده ازٔ ساندويج و جييس و يفك، بهتر از تنقالاتى هميجون كردو و بادام است.

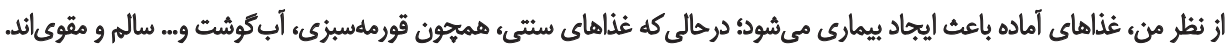

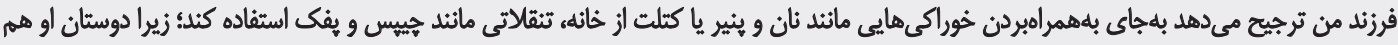

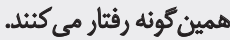

مصرف غذاهاى فورى، مائند سوسيس و كالباس در خانواده ما زياد است؛ بهميندليل، فرزندانم نيز بهطورمرتب از اين غذاهاى أماده مصرف مى كنند. بهدليل اينكه خودم سوسيس و كالباس و همبركر را دوست دارم و مصرف مى كنه، براي مصرف اين كونه غذاها با فرزنلانم مخالفت نمى كنه.

كاهى ييش مى آيد كه ناهار يا شام را دير مىخورم يا اينكه اصلاً نمىخورم.

معمولاً صبحها كه از خواب بيدار مي شومه، صبحانه نمى خورم.

در خانواده ما، مصرف غذاهاى فورى، مثل سوسيس، كالباس، كنسروها و غذاهاى سرخكردنى زياد است.

در خانواده ما، مصرف غذاهاى دريايى، مائند ماهى كمم أست.

كاهى يبش از حد اشتمهايم غذا مى خورم.

باوجودائكه هميشه كفته مى شود غذاهاى آماده و سخشده خوب نيست؛ اما من به غذاهاى سرخشده بيشتر از غذاهاى بخته علاقه دارم. ماهي با ديكر اعضاى خائواده، در أثتخاب غناها الختلاف سليقه داريم.

از نظر من، غُذاهاي جرب خوشمزهترند.

از نظظر من، غذاهاي شور خوشمزهترند.

\section{دانش تُغذيها|ى}

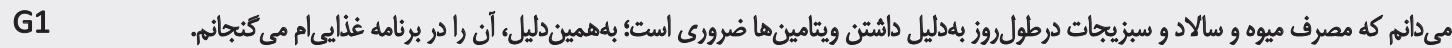

G2

مى دائم كه مصرف ماست بهدليل تأمين كلسيم و بروتئين براى بلن لازم است؛ بههمين دليل، همراه با وعدههاي غذاييى ماست مصرف مي كنم.

G3

مى دانم كه بايلد بيشترين نوع موادغذايي كه مصرف مى كنه، از كروه غلاته نانه برنج و ماكارونى باشد؛ هراكه مهمترين منبع ثأمين اثرزى هستند.

G4

هى دانم كه روغن نباتى جامد، براى قلب مضر است و تاحدممكن از آن كم استفاده مى كنم.

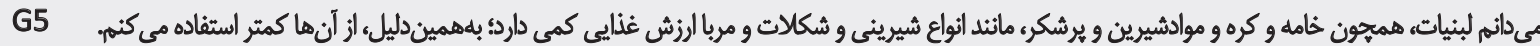

G6

ميدانم كه نمك زياد، فشارخون را افزايش ميدهل؛ بنابراينء سرسفره به غذا نمك اضافه نمي كنمه.

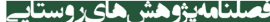

فرهنك غذايى و دانش تغذيهاى بر سطح نامنى غذايى خانوار،

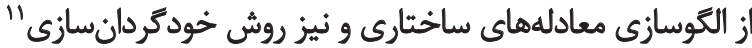

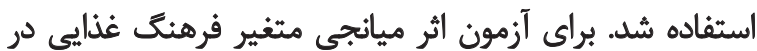

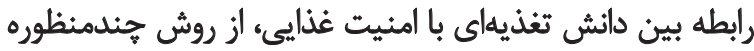

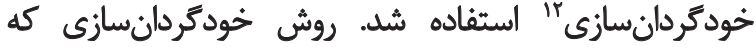

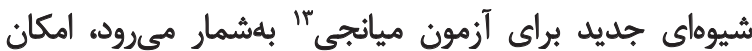

11. Bootstrapping

12. Bootstrap

13. Mediation test

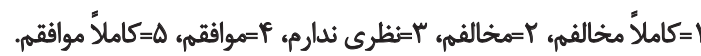

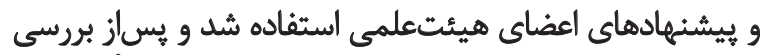

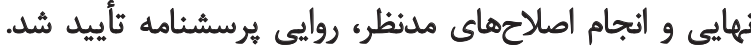

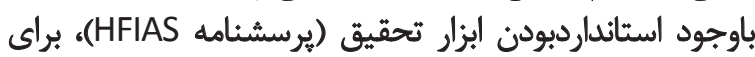

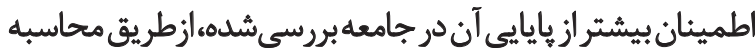

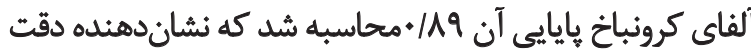

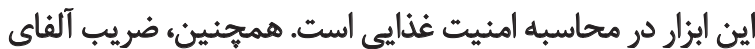

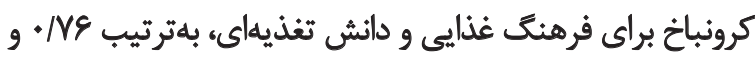

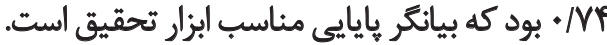
بهمنظور تجزيهوتحليل دادهاي كردآورىشده و بررسى اثر 


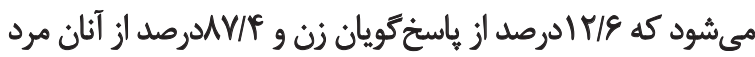

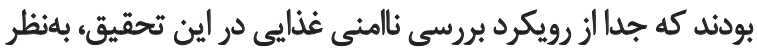

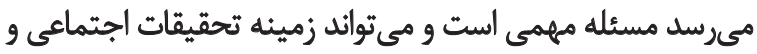
حتى اقتصادى و منطقهاى ديكرى قرار گيرد.

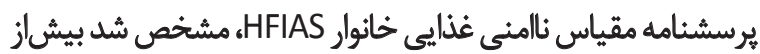

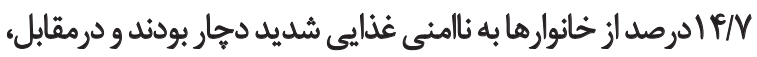

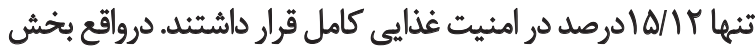

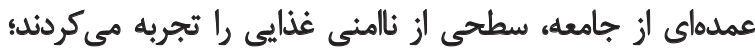

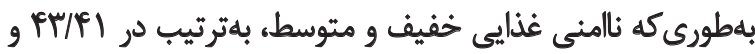
FiVFF بلهمنظور آزمون فرضيههاى اصلى ثئوهش در زمينه تأثير

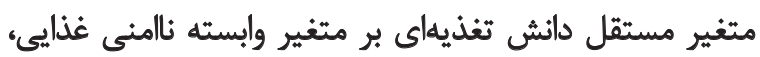

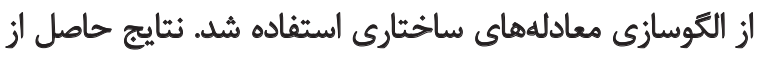

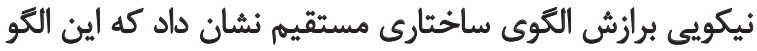

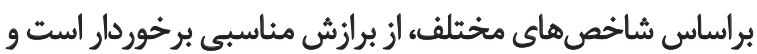
مي تواند مبناى تحليل قرار بكيرد (جدول شماره f). تصوير شماره ا و جدول شماره هـ نتايج برآورد الكوى ساختارى با سنجش سطح نامنى غذايى باتوجهبه امتياز بهدستآمده آزئل

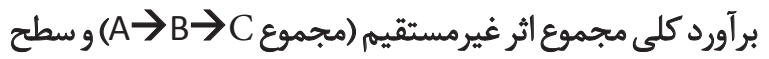

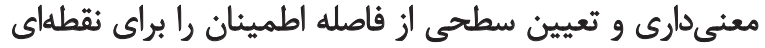

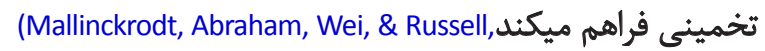

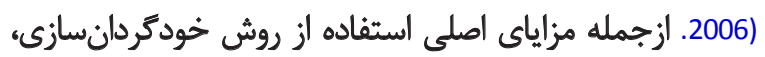

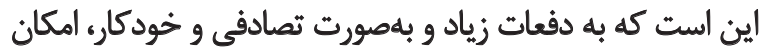

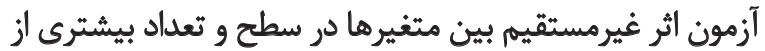

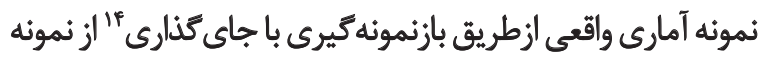

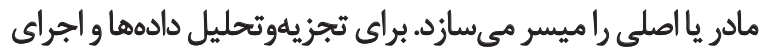

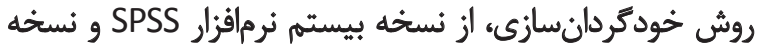

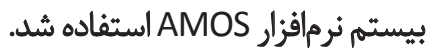

يافتهها

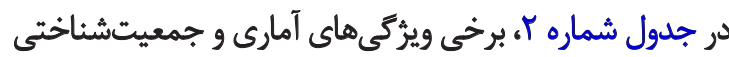

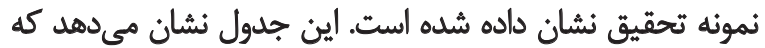

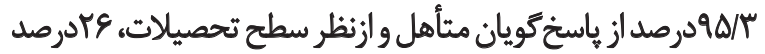

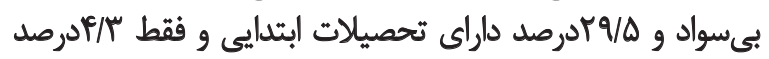

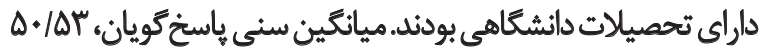

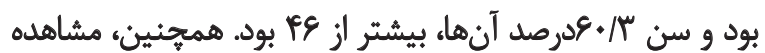

14. Resampling with replacement

جدول ז. توزيع فراواني ياسخ كويان براساس ويُّكي هاي فردى

\begin{tabular}{|c|c|c|c|}
\hline درصد & فوراوانى & هتغير & \\
\hline$A V / F$ & Mr & هرد & \multirow{3}{*}{ جنسيت } \\
\hline & & & \\
\hline Ir/S & $M$ & زن & \\
\hline$P / V$ & ir & مجرد & \multirow{2}{*}{ وضعيت تأهل } \\
\hline $9 \Delta / \%$ & MTT & مثتأهل & \\
\hline re & eq & ل بي سواد & \multirow{6}{*}{ سطح تحصيلات } \\
\hline$r q / \Delta$ & VA & ابتدايع & \\
\hline$r \cdot / q$ & هr & راهنمايى & \\
\hline$S / V$ & IV & دييرستان & \\
\hline $\mathrm{Ir/S}$ & M & دييلم & \\
\hline$e / N$ & 11 & تحصيلات دانشكاهي & \\
\hline $\mathrm{IV} / \mathrm{T}$ & pe & هrot & \multirow{5}{*}{ 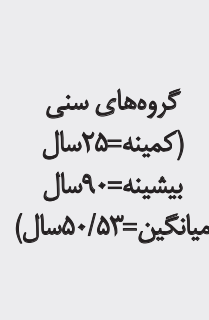 } \\
\hline$r T / F$ & $\Delta V$ & عr تا ه بال & \\
\hline re & ge & عن تا ه سال & \\
\hline $\mathrm{r} / \mathrm{r}$ & $\Delta f$ & عه تا هاء سال & \\
\hline ir & Tr & و\& سال و بيشتر & \\
\hline
\end{tabular}

encing 
جدول ץ. توزيع فراوائى ياسخ گويان براساس سطح نامنى غذايى

\begin{tabular}{|c|c|c|}
\hline درصد & فراواتى & سطح نامنى غذايي \\
\hline 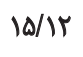 & rq & داراى امثيت غذايى (• تا م) \\
\hline 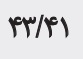 & 114 & 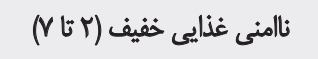 \\
\hline$T \& / M^{e}$ & eq & 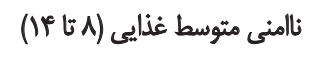 \\
\hline $1 f / M$ & ra & ثالمنى غذايي شديد (ها و بيشتر) \\
\hline
\end{tabular}

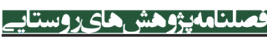

جدول F. نتايج ميزان انطباث الكّوى ساختارى مستقيه با شاخصهاى برازش

\begin{tabular}{|c|c|c|c|c|c|c|}
\hline RMSEA & TLI & CFI & IFI & GFI & $x^{2} / d f$ & شاخص \\
\hline$\leq+1+1$ & $\cdot / q . \leq$ & $\cdot 19 . \leq$ & $\cdot / 9 \cdot \leq$ & $\cdot / 9 . \leq$ & $\leq \Delta$ & معيار ييشنهادشهد \\
\hline .1 .98 &.$/ 9 V^{2}$ & ./RA &.$/ 410$ &.$/ 9 r^{2}$ & $r / . q r$ & مقدار كزأرش شده \\
\hline
\end{tabular}

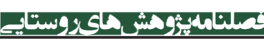

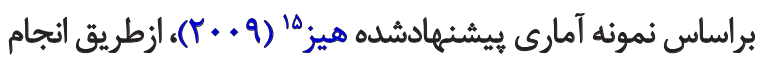

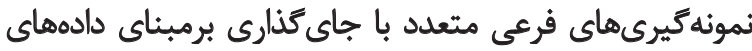

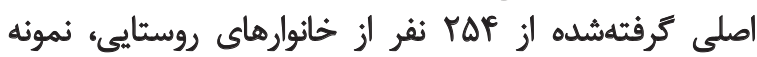

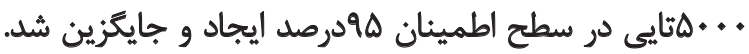

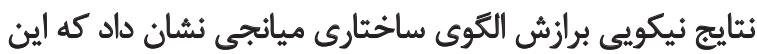

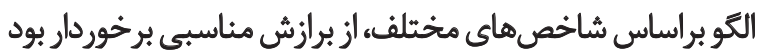

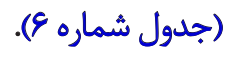

نتايج مئدرج در جدول شماره V در زمينه اجراي روش

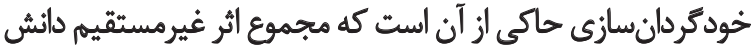

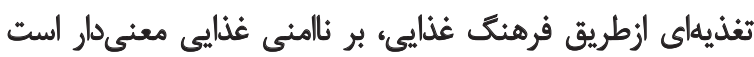

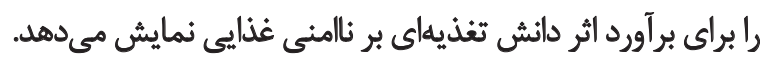

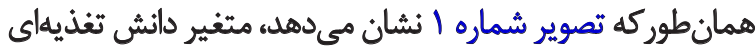

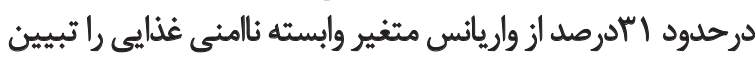

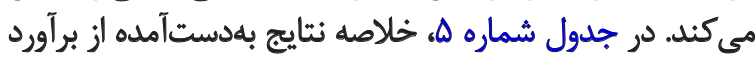

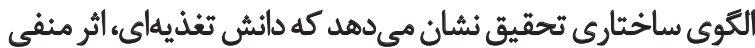

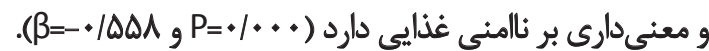

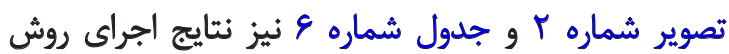

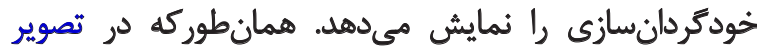

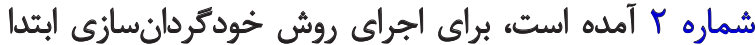

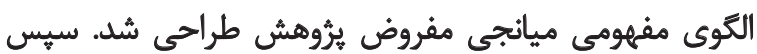

Chi-square $(\mathrm{df})=25.114$ (12); $P$ value $=.014$;

Relative Chi-sq = 2.093;

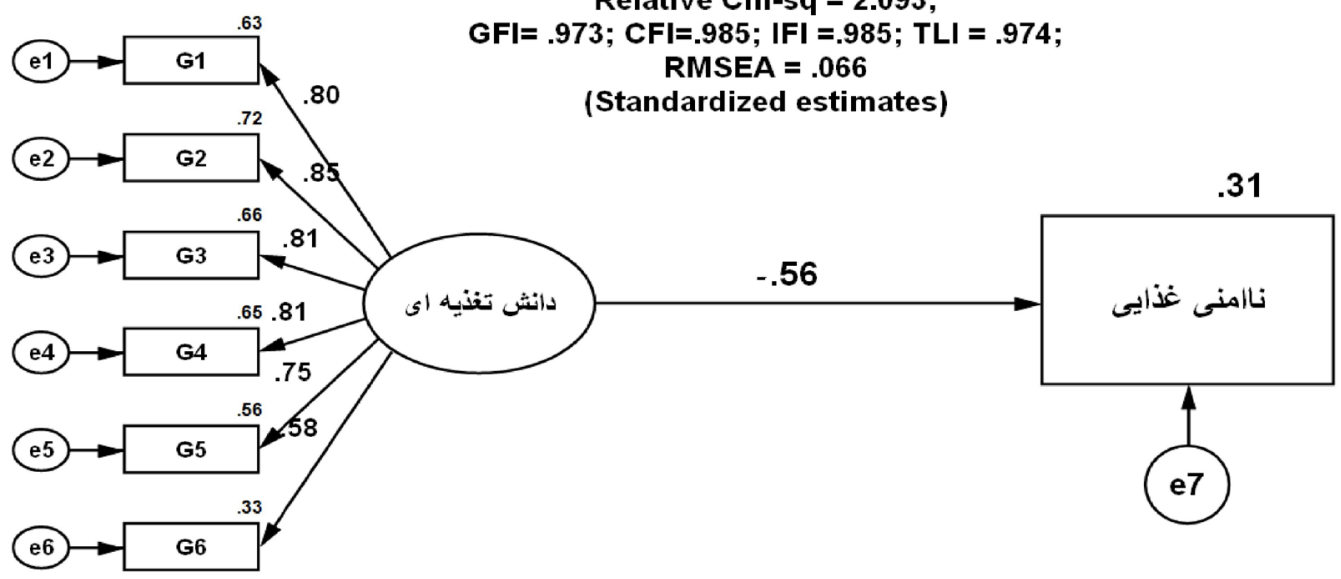


جدول ه. ضرايب ركرسيونى بين متغيرهاى دانش تغذيهاي و نامنى غذايى

\begin{tabular}{|c|c|c|c|c|c|}
\hline سطح معنى دارى & نسبت بحرانى & ضريب اسثاندارد & خطاى استاندارد & مقدار غير اسثاندارد & وابطه ابط \\
\hline$+\infty$ & $-q / / M$ & $-+/ \Delta \Delta \Lambda$ &.$/ P n$ & $-F / M T E$ & دانش تغذيهاى ـ نالمثى غذايى \\
\hline
\end{tabular}

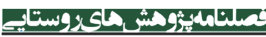

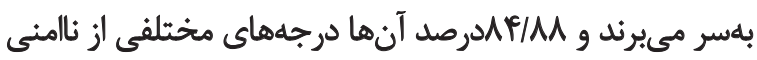
غذايى را تجربه مى كنند. برايناساس، بهنظر مئرسد كه شئ شيوع نامنى غذايى در مناطق روستايى شهرستان كرمائشاه، تاحدودى دئي

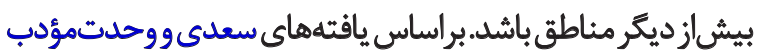

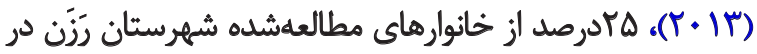

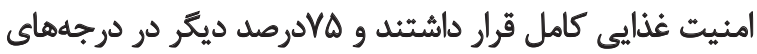

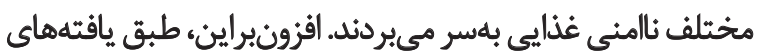

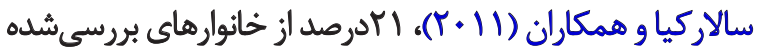

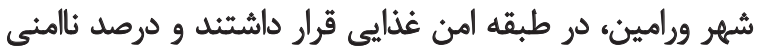

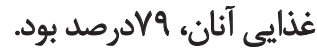

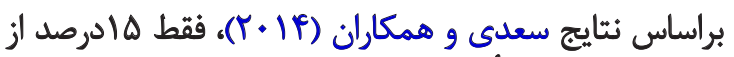

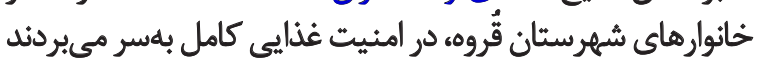
و بقيه درجهاي مختلفي از نامنى غذائي داشتيند. در مطالعهاي
غذائ

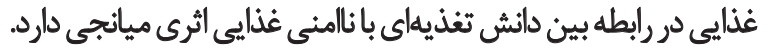
در جدول شماره Aه، آثار مستقيهم و غير مستقيم ازطريق فرهنى

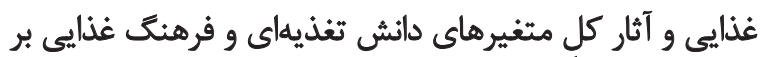

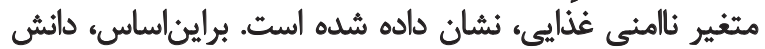

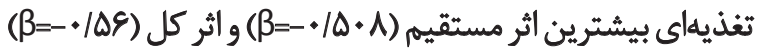
رابر نامنى غذايى روستاييان دارد.

\section{ى 1 S.}

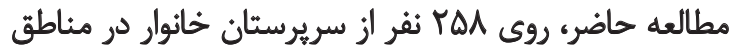

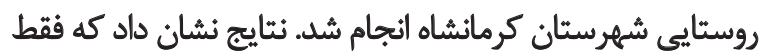

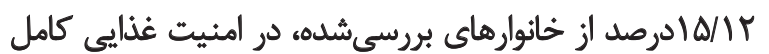

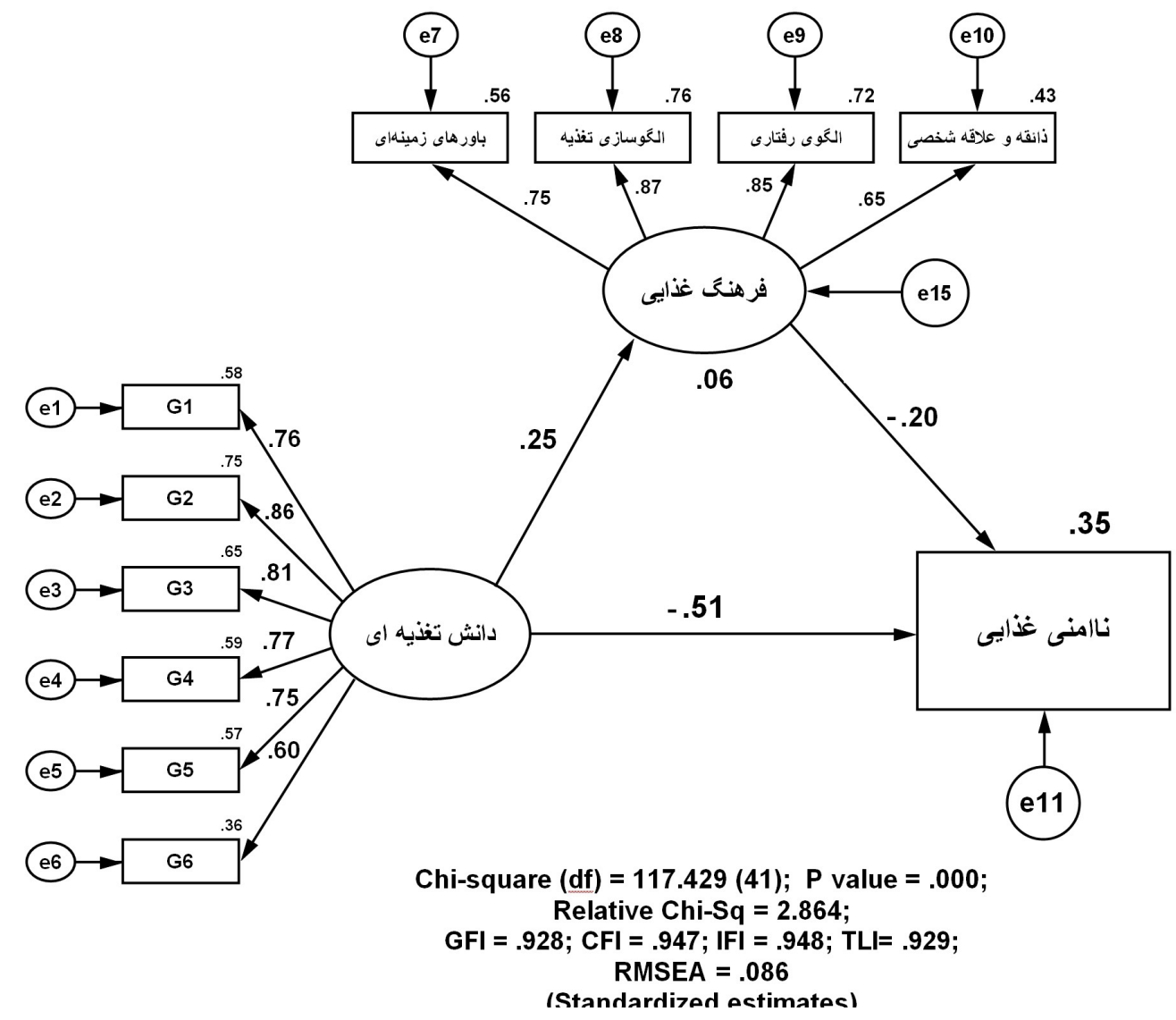


جدول و. نتايج ميزان انطباق الكوى ساختارى ميانجى با شاخصهاي برازش

\begin{tabular}{|c|c|c|c|c|c|c|}
\hline RMSEA & RMSEA & TLI & CFI & IFI & GFI & $x^{2} / d f$ \\
\hline$\leq * 1 \cdot 1$ & $\cdot / q \cdot \leq$ & $\cdot / 9 . \leq$ & $\cdot / 9 \cdot \leq$ & $\cdot / 9 . \leq$ & $\leq \Delta$ & معيار ييشنئهادشده \\
\hline .1 .18 &.$/ 979$ & - /apa & - /9ra & ./9TA & T/AOP & همدار تززارشيده \\
\hline
\end{tabular}

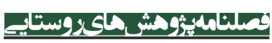

جدول \. اثر دانش تغذيهاى ازطريق فرهنك غذائى بر نامنى غذايى (روش خودكردانسازي)

\begin{tabular}{|c|c|c|c|c|c|}
\hline \multicolumn{3}{|c|}{ 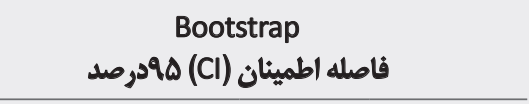 } & \multirow{2}{*}{ خطائ استائدارد } & \multirow{2}{*}{ غيثانداردشيره } & \multirow{2}{*}{ رابطه } \\
\hline 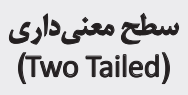 & كران بالا & كران نايين & & & \\
\hline $.1+.1$ & -.1 .94 &.$-|\cdot r|$ & $\cdot 1 \cdot 11$ &.$-|\cdot \Delta|$ & دانش تغذيلهاى ـ فرهنك غذايى ـ نالمنى غذايى \\
\hline
\end{tabular}

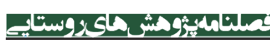

جدول A. تفكيك آثار مستقيم و غيرمستقيم ازطريق فرهنَ غذايى بر نامنى غذايى براساس الكّوى ميانجى

\begin{tabular}{|c|c|c|c|}
\hline \multicolumn{3}{|c|}{ آثار استانداردشده } & \multirow[b]{2}{*}{ 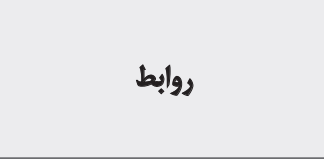 } \\
\hline 5 & (ازطريق فرهئي غذايقيى) & مستقيم & \\
\hline . MAT & - & . /TAT & دانش تغذيهاى ـ ـرهنك غذايى \\
\hline$-\cdot / r \cdot r$ & - & $-\cdot / r \cdot r$ & فرهنى غنايع ؟ نامنى غذايع \\
\hline $\begin{array}{c}-(\cdot / \Delta \cdot A+\cdot 1 \cdot \Delta 1) \\
=-\cdot 1 \Delta 5\end{array}$ & $\begin{array}{c}(\cdot / r \Delta r X-\cdot / r \cdot Y) \\
=-\cdot / \cdot \Delta 1\end{array}$ & $-+10 \cdot 1$ & دانش تعذئياى ـ نالمنى غذايي \\
\hline
\end{tabular}

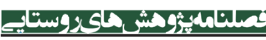

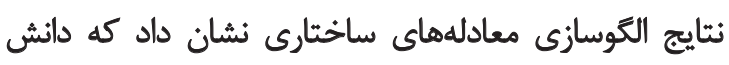

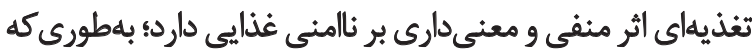

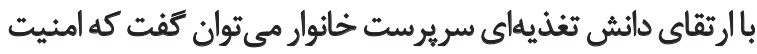

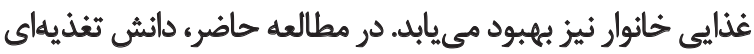

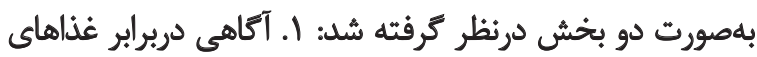

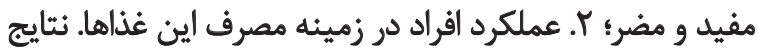

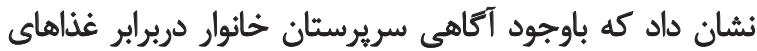

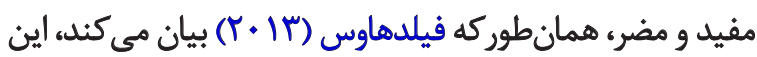

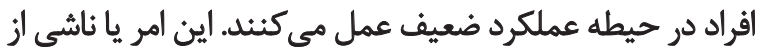

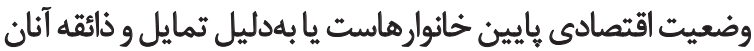

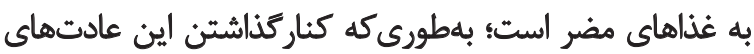

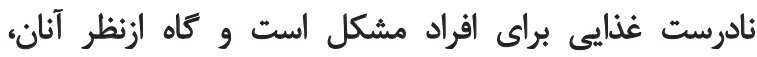

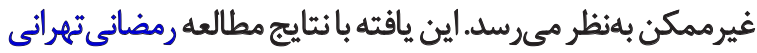

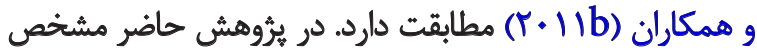

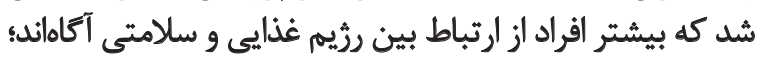

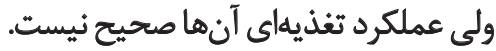

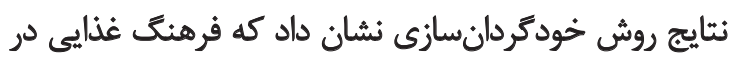

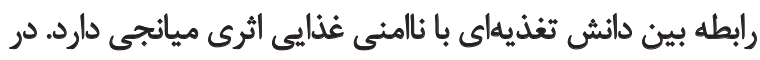

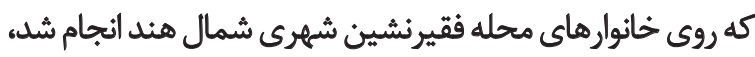

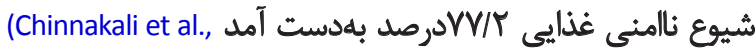
(2014. ميزان شيوع نالمنى در كويمباتور كشور هند در خائوارهاي

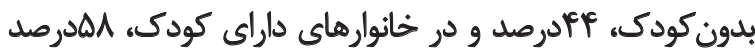

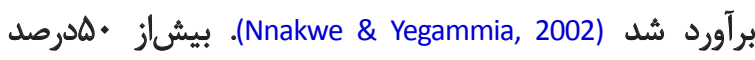

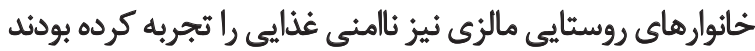

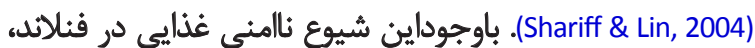
فقط ه/ هدرصد بود (Sarlio-Lahteenkorva, 2001).

دليل اختلاف درنتايج رامىتوان به تفاوت در شرايط جغرافيايى،

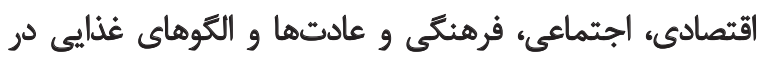

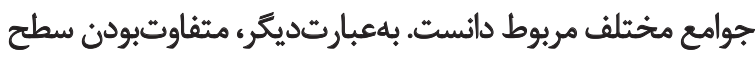

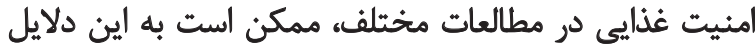
باشد: ثفاوت در روش تعيين وضعيت امنيت (نالمني) غنايي و

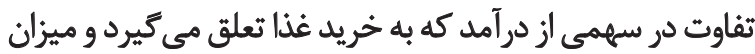
كمكهايي كه در جوامع به خانوارهاي كمدر آمد ارائه مي دئودي

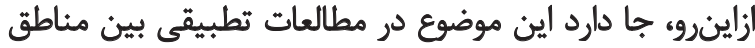
مختلف مدنظر قرار كيرد. 
هروهش حاضر، فرهنَّ غذايى در قالب هجهار زيرطبقه باورهاى

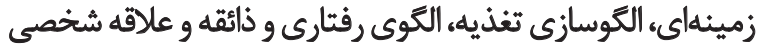

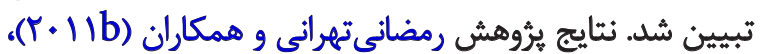

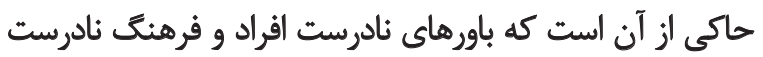

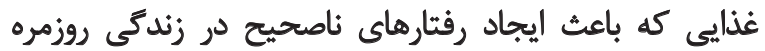

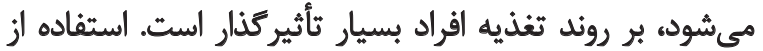

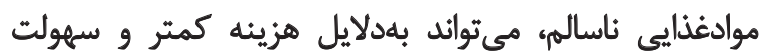

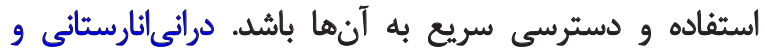

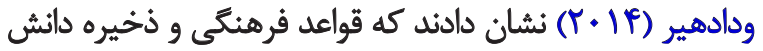

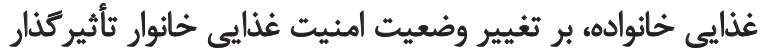

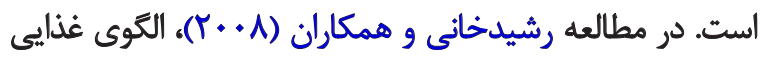

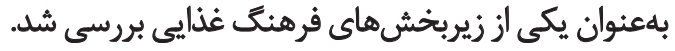
نتايج تحقيق حاضر نشان مي دهد كه رفتارهاي غذايى افراد

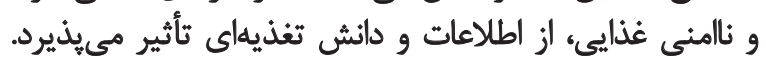

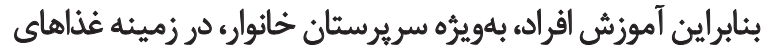

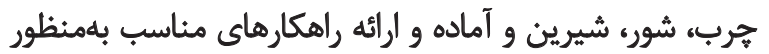

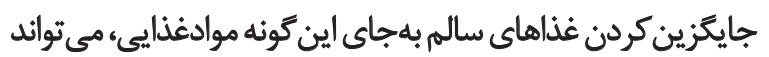

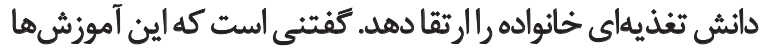

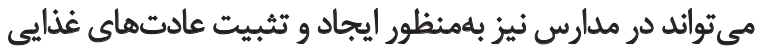

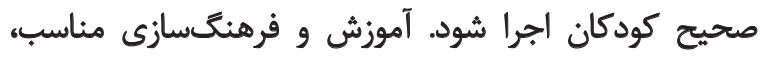

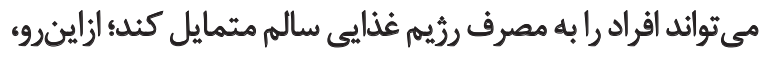

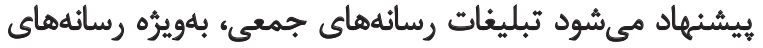

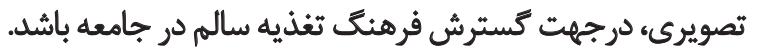

$$
\text { تشكر وقدرهانى }
$$

اين مقاله از باياننامه كارشناسى ارشد خانم زهره رستان رستميان

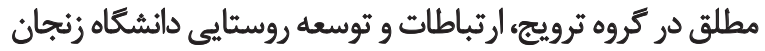

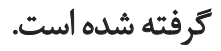




\section{References}

Ballard, T. J., Kepple, A. W., \& Cafiero, C. (2013). The food insecurity experience scale: Development of a global standard for monitoring hunger worldwide. Rome: Food and Agriculture Organization.

Bartlett, J. E., Kotrlik, J. W., \& Higgins, C. C. (2001). Organizational research: Determining appropriate sample size in survey research. Information Technology, Learning, and Performance Journal, 19(1), 43-50.

Chinnakali, P., Upadhyay, R. P., Shokeen, D., Singh, K., Kaur, M. Singh A. K., et al. (2014). Prevalence of household-level food insecurity and its determinants in an urban resettlement colony in North India. Journal of Health, Population, and Nutrition. 32(2), 227236. PMCID: PMC4216959

Coates, J., Frongillo, E. A., Rogers, B. L., Webb, P., Wilde, P. E., \& Houser, R. (2006). Commonalities in the experience of household food insecurity across cultures: What are measures missing? Journal of Nutrition, 136(5), 1438S-1448S.

Coates, J., Swindale, A., \& Bilinsky, P. (2007). Household Food Insecurity Access Scale (HFIAS) for measurement of food Access: Indicator guide. Version 3. Washington, D. C.: Food and Nutrition Technical Assistance (FANTA) Publication

Darrani Anarestani, M., \& Vedadhir, A. (2014). [The role of knowledge and culture of household heads on the food security (Persian)]. Paper presented at The Third National Conference on Food Security, Savadkuh, Iran, 26-27 February 2014

Dastgiri, S., Mahboob, S., Tutunchi, H. \& Ostadrahimi, A. (2006). [Determinants of food insecurity: A cross - sectional study in Tabriz (Persian)]. Journal of Ardabil University of Medical Sciences, 6(3), 233-239.

Fieldhouse, P. (2013). Food and nutrition: Customs and culture. Berlin: Springer.

Food and Agriculture Organization (FAO). (2015). The state of food security in the world. New York: Food and Agriculture Organization.

Froutanfar, L. (2013). [Assessing food security and its influencing factors in the rural households of Baladarband Rural District (Persian)] [MSc. thesis]. Kermanshah: University of Razi.

Furness, B. W., Simon, P. A., Wold, C. M., \& Asarian-Anderson, J. (2004). Prevalence and predictors of food insecurity among lowincome households in Los Angeles County. Public Health Nutrition, 7(6), 791-794. doi:10.1079/phn2004608

Ghassemi, H., Harrison, G., \& Mohammad, K. (2002). An Accelerated Nutrition transition in Iran. Public Health Nutritions, 5 (1A), 149-155. https://doi.org/10.1079/PHN2001287

Hayes, A. F. (2009). Beyond baron and Kenny: Statistical mediation analysis in the new millennium. Communication Monographs, 76(4), 408-420. doi: 10.1080/03637750903310360

Hezarjaribi, J., \& Alizadeh Aghdam, M. (2013). [The study of the impact of socio-economic capital upon nutrition among the citizens of Tabriz (Persian)]. Jame'e Shenasi-e Eghtesadi va Tose'e, 1(2), 195-220.

Jafari Sani, M., \& Bakhshoudeh, M. (2008). [Examining the spatial distribution of poverty and food insecurity in rural and urban households to separate provinces in Iran (Persian)]. Agricultural Economic and Development, 16(61), 103-123.
Loring, P. A., \& Gerlach, S. C. (2009). Food, culture, and human health in Alaska: An integrative health approach to food security Environmental Science \& Policy, 12(4), 466-478. doi: 10.1016/j.envsci.2008.10.006

Mallinckrodt, B., Abraham, W. T., Wei, M., \& Russell, D. W. (2006) Advances in testing the statistical significance of mediation effects. Journal of Counseling Psychology, 53(3), 372-378. doi: 10.1037/00220167.53.3.372

Mobini Dehkordi, A. (2005). [New Approach to choose the best option food security strategy for Islamic Republic of Iran (Persian)] Agricultural Economic and Development, 13(51), 15-32.

Molnar, J. J. (1999). Sound Policies for Food Security: The role of culture and social organization. Applied Economic Perspectives and Policy, 21(2), 489-498. doi:10.2307/1349893

Namakin, K., Moasheri, N., \& Khosravi, S. (2012). [Studying Birjand girls' secondary school students' nutritional pattern (Persian)] Modern Care Journal, 9(3), 264-272.

Nnakwe, N., \& Yegammia, C. (2002). Prevalence of food insecurity among households with children in Coimbatore, India. Nutrition Research, 22(9), 1009-1016. doi: 10.1016/s0271-5317(02)00419-0

Poorghasem, F., Pourjavid, S., \& Alibeigi, A. (2013). [Factors affecting nutritional status of rural households in Kermanshah township (Persian)]. Journal of Rural Research, 4(2), 347-364.

Power, E. M. (2008). Conceptualizing food security for aborigina people in Canada. Canadian Journal of Public Health, 99(2), 95-97.

Ramezani Tehrani, F., Farahmand, M., Amiri, P., Ghanbarian, A., \& Azizi, F. (2011a). [Healthy nutrition barriers: Perception of adults male (Persian)]. Payesh, 11(5), 725-735.

Ramezani Tehrani, F., Farahmand, M., Amiri, P., Paikari, N., \& Azizi, F. (2011b). [Women's perception regarding to healthy nutrition Inhibitors: A qualitative research in Tehran lipid and glucose study (TLGS) (Persian)]. Iranian Journal of Endocrinology and Metabolism, 13(1), 48-57.

Rashidkhani, B., Rezazadeh, A., Omidvar N., Houshiar Rad, N., \& Setayeshgar, A. (2008). [Relationships of major dietary patterns and their association with socioeconomic and demographic factors in 20-50 year- old women in the north of Tehran (Persian)] Iranian Journal of Nutrition Sciences \& Food Technology, 3(2), 1-12.

Reichwage, M. (2010). Food security among households in northern Nigeria: Descriptive analysis [MSc. thesis]. Atlanta: Emory University.

Riyahi, M. (2005). [A comparative study of gender differences and dietary habits a survey of the Iranian and Indian students (Persian)]. Women's studies (Sociological \& Psychological), 3(2), 97-126

Rostami, F., Shahmoradi, M., \& Baghaie, S. (2015). [Study of factors affecting on rural households food security case study: Karnachy village in Kermanshah County (Persian)]. Iranian Journal of Agricultural Economics and Development, 45(4), 725-737.

Rostamian Motlagh, Z. (2015). [Estimating food security in the rural areas of the Kermanshah province and investigating its influencing factors (Persian)] [MSc. thesis]. Zanjan: University of Zanjan.

Saadi, H., \& Vahdat Moaddab, H. (2013). [Assessment of femaleheaded household's food security and the affecting factors; Case Study: Women in Razan City (Persian)]. Women in Development E Politics, 11(3), 411-426 
Saadi, H., Azizi, M., \& Azami, M. (2014). [Extension education and food security of farmer households (Case study: Qorveh County, Kurdistan Province) (Persian)]. Iranian Journal of Agricultural Economics and Development, 45(3), 483-499.

Salarkia, N., Abdollahi, M., Amini, M., \& Eslami Amirabadi, M. (2011). [Validation and use of the HFIAS questionnaire for measuring household food insecurity in Varamin (Persian)]. Iranian Journal of Endocrinology and Metabolism, 13(4), 374-383.

Sarlio-Lahteenkorva, S., \& Lahelma, E. (2001). Food insecurity is associated with past and present economic disadvantage and body mass index. Journal of Nutrition, 131(11), 2880-2884.

Shariff, Z. M., \& Lin, K. G. (2004). Indicators and nutritional outcomes of household food insecurity among a sample of rural Malaysian women. Pakistan Journal of Nutrition, 3(1), 50-55. doi: $10.3923 /$ pjn.2004.50.55

Willows, N. D., Veugelers, P., Raine, K., \& Kuhle, S. (2008). Prevalence and sociodemographic risk factors related to household food security in Aboriginal peoples in Canada. Public Health Nutrition, 12(8), 1150-1156. doi: 10.1017/s1368980008004345 